\title{
REVIEW
}

\section{PHYSICISTS, STAMP COLLECTORS, HUMAN MOBILITY FORECASTERS ${ }^{1}$}

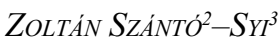

\begin{abstract}
Albert-László Barabási: Villanások - a jövő kiszámítható. Nyitott Könyvmühely, Budapest, 2010.

Albert-László Barabási: Bursts. The Hidden Pattern Behind Everything We Do, Dutton Adult, New York, 2010.
\end{abstract}

One of the two reviewers studied in high school to be a physicist. In the end, he became something else, but he never lost his awe of physics. The other reviewer never intended to become a physicist, but he sometimes asks himself why he didn't become one. Today, they are both sociologists who practice their science on an action theory basis and believe that regularities exist in the world of social actions which can be perceived, understood, explained - and even used for making predictions.

Albert-László Barabási, a world-renowned physicist, wrote a book in 2010 about the regularities of human behavior. Barabási asks the often-repeated question: "Is human behavior predictable?" Many believe that, since we cannot describe human actions with precision, we also cannot make predictive statements with reference to them. According to Barabási, however, "the future is foreseeable". Who is right? Barabási's answer is clear: we should believe the one who is doing the counting - the physicist.

1 The Hungarian version of this article was published in Budapest Review of Books (BUKSZ, 2010/Fall).

2 Zoltán Szántó is professor of sociology at the Corvinus University of Budapest; e-mail: zoltan. szanto@uni-corvinus.hu

3 SYI (István Szakadát) is associate professor of sociology at the Budapest University of Technology and Economics; e-mail: i@syi.hu. 
"Biologists confronted with the mindboggling complexity of a cell, brain scientists humbled by the miracle of our neural circuitry, and social scientists and economists at a loss before the labyrinth of social and economic processes, from economic bubbles to crises, have often argued that fundamental laws may not exist in their field. As such, many judge the physicist's insistent search for universal laws to be misguided at best and, at worst, bound for failure" (p.50).

Thus, biologists' minds are boggled, brain scientists are humbled and social scientists are at a loss. They give up the fight, since, in their field, they can never "hope to describe human behavior as accurately as we are able to describe the material world" (p.51), or to identify regularities that are generalizable. And then come the physicists who find universal laws. They are able to do this because, in this digital age, we are capable of gathering and analyzing an immense amount of information about human behavior. At the same time, they also answer another long-disputed question: is there one or two kinds of culture, one or two kinds of science? Barabási quotes Rutherford here: "in science, there is only physics, all the rest is stamp collecting" (p.50).

Well...that is a bit bold...a bit provocative...perhaps even a bit unfounded. The appropriate response here is to be somewhat offended.

And yet we are not. We are not offended, because, in essence, we agree with this sharp opinion. Barabási is right: we have, indeed, arrived at the beginning of a new era. And from here on, we, social scientists (and other stamp collectors), must strive towards thinking and working together. Because the foundations are solid, but the direction is not always optimal. Although Barabási is not a social scientist, without knowing him there is no point in doing social science. We do not necessarily have to follow him, but we must understand him.

\section{ABOUT THE BOOK - IN MORE DETAIL}

Bursts will probably have the success of Barabási's previous book - Linked. How Everything Is Connected to Everything Else, and What it Means for Science, Business, and Everyday Life ${ }^{4}$. In the latter work, he summarizes the findings of his research on network theory in a light, easy-to-understand style. His analysis centers on scale-free networks, with discussions on their unique topology, structure and wide distribution. Earlier approaches assumed that

4 Penguin Group, 2002. The book became a real scientific bestseller. 
different types of networks (physical, chemical, biological, and social) came into existence in an accidental manner. Barabási, however, was among the first to recognize (in the nineties) that this is not so: not only can strict and universal regularities often be discovered behind the structure of various types of networks, they also show the same power law pattern. In his new book, Barabási takes his earlier ideas in an exciting and controversial (especially in social science circles) new direction.

Barabási begins Bursts with the aforementioned - for social scientists, rather provocative - question, reminiscent of the spirit of classic positivism: "Are our actions governed by rules and mechanisms that might, in their simplicity, match the predictive power of Newton's law of gravitation?" (p.7). He answers with a definite yes and then articulates his final conclusion:

"When we break our lives down to numbers, formulas, and algorithms, we are actually much more similar to one another than we might be ready to admit. Granted, you do what you think is best for you, and you do it when you can and when it is the most convenient for you. You may live in L.A. and I in Boston, you may be Asian and I Hungarian, you may run a restaurant while I do research, teach, and occasionally write books. These differences do matter; no one has ever really questioned that. But if we focus on our action and their timing, we see patterns that are not unique to you and me. They pertain to billions. We are simultaneously bursty and quite regular. Apparently random yet deeply predictable. Sure, some events we face are quite haphazard. But the way we cruise through them is universal" (p.254).

In order to understand his thinking, we will briefly present the scientific findings, empirical studies, theoretical investigations, and everyday observations based on which Barabási attempts to lay the foundations for a new scientific field, which he refers to as human dynamics.

At the beginning of the book, he writes: "algorithms built in my lab to discover how predictable we are were tested on millions of individuals and failed only once. His name was Hassan. Hassan Elani, to be precise." (p.1). The story of Elani, the media artist, runs through the book. Barabási's aim is to illustrate how he is a true exception in that he does not follow regular and predictable patterns shown by the mobility of the majority of people. Or, to put it more precisely, he is an example for what bursts represent within the phenomenon of human mobility. His unique story, whose interesting details keep resurfacing in subsequent chapters of the book, demonstrates the fact that the occurrence of a few individuals of exceptional mobility is just as regular as that of the masses which show a large degree of predictability in 
their movement. This is so even if exceptions are rare - or precisely because they are rare. This connection is illustrated by Barabási through the simple-tograsp concept of "the larger, the fewer" (p.177), which, in a specific scenario, means that there are few who travel often and to many different destinations (like Elani) and many who travel rarely and to few destinations. Human mobility can be precisely described and predicted with the help of the power law.

In the book, we encounter another burst taken from Hungarian history which is probably unfamiliar to most non-Hungarian readers. The evennumbered chapters of the volume - at first take, in a surprising manner - give a historically precise account of the events leading up to and surrounding the peasant revolt led by György Dózsa in 1514. These chapters provide an overall picture of this episode in history, from the failure of Tamás Bakócz's papal ambitions, through the formulation of the ranks of the crusaders' army, István Telegdi's prediction, the slaughter at Apátfalva, and the Battles of Temesvár and Kolozsvár, to the final reckoning and the seating of Dózsa on a throne of smoldering iron. The author himself poses the justly-asked question: "What is György Székely ${ }^{5}[\ldots]$ doing in a book about human dynamics?" (p.254). He reasons that, in addition to being an eloquent testimony to the significance of bursts in history, this detailed case study is also interesting in its train of thought because events unfolded precisely as foreseen by Count Telegdi. It is thus that the question arises: can historical events be predicted?

Barabási argues against Karl Popper's widely accepted view according to which any attempt at predicting social, economical and political processes will necessarily run into problems of principle and points out that "there is no solid proof that social systems are impossible to predict" (p.256). He writes:

"But no physicist has ever successfully predicted the trajectory of 1023 molecules in a gas, either, and that hasn't stopped us from predicting the gas's pressure and temperature-arguably far more important than the trajectory of each individual molecule. The same is true for human dynamics. Our deep-rooted unpredictability does not need to bubble up at the level of society. If we carefully distinguish the random from the predictable, we might be able to foresee many features of the social fabric" (ibid.).

In his discussion, he also refers to another analogy. In our current times, short-term (3-day) meteorological forecasts are nearly 95\% accurate. In contrast, at the beginning of the twentieth century, the pioneer of weather

5 Barabási refers to Dózsa as György Székely. 
forecasting, Lewis Fry Richardson, tried to predict daily weather conditions by finding a day of similar weather from the past, and taking the conditions of the next day to indicate what the weather would be like on the day following his forecast. This technique of weather forecasting took immense amounts of time and effort and was very inaccurate. According to Barabási, Richardson's failure was an indication not of the impossibility of weather forecasting, but of the limitations of weather data and computation capacities available at the time. There is no notable difference in the current methodology for predicting weather conditions, but we now have a significantly larger amount of data to analyze and a greatly increased capacity for making calculations.

The question here is whether Barabási is justified in making the assumption that - thanks to an accumulation of databases and the increasingly more developed techniques of computer analysis - a turn has occurred in recent years which now make it possible to more effectively uncover the rules of human dynamics and, thus, better predict human mobility. In his view, this indeed is the case:

"today just about everything we do leaves digital breadcrumbs in some database. Our e-mails are preserved in the log files of our e-mail provider; time-stamped information about our phone conversations sits in our phone company's vast hard drives; when, where, and what we shop for, our taste, and our ability to pay for it is catalogued by our credit card provider; all our Web pages, MySpace and Facebook profiles, and blogs are saved and crosslisted in multiple servers; our momentary whereabouts are available to our mobile phone provider; our face and fashion is remembered by countless surveillance cameras installed everywhere, from shopping malls to street corners. [...] our life, with minute resolution, can be pieced together from these mushrooming databases. [...] It also creates historic opportunity, however, offering for the first time unbiased data of unparalleled detail on the behavior of not one, but millions of individuals. In the past few years, these databases have found their way into a variety of research labs, allowing computer scientists, physicists, mathematicians, sociologists, psychologists, and economists to pore over them with the help of powerful computers and a wide array of novel technologies. Their conclusions are breathtaking; they provide convincing evidence that most of our actions are driven by laws, patterns, mechanisms that in reproducibility and predictive power rival those encountered in the natural sciences" (p.10). 
In the odd-numbered chapters of the book, Barabási shows how - and based on what research results - he arrived at the above conclusions, in an easy-toread, accessible style (with references and sources listed in the notes at the end of the book $)^{6}$. From this point on, to the extent allowed by the scope of this review, we will examine these research findings.

(1) Researchers who study the spread of human-to-human contagious diseases need to have an understanding of how people move and interact. The website www.wheresgeorge.com - which, in the form of a uniquely formulated public game, tracks millions of dollar bills throughout the United States proved to be a goldmine for coming by this type of information. It was based on analysis of the movement of these banknotes that a hypothesis for the random nature of human mobility was established by natural scientists examining the phenomenon. According to this theory, banknotes move in a random manner, and, since they go where people take them, it follows that humans also move randomly. The findings were first published by a young German physician, Dirk Brockmann, and his colleagues in 2006 in Nature, under the title "The Scaling Law of Human Travel", rousing considerable attention in scientific circles. The expectations in the hypothesis seemed to correspond with the superdiffusive law worked out by Albert Einstein and Baptiste Perrin, which predict the dynamics of tiny particles in water (and 'incidentally' also proved the existence of atoms for which Perrin received the Nobel Prize in physics in 1926). The movement of a few dollar bills, however, did not fit this random pattern, and, in fact, followed a trajectory that was completely different from the expected diffusion pattern. While the hypothesis about the randomness of human mobility seemed to be crumbling, the inquiry itself set off an avalanche and served as the point of departure for numerous further research initiatives.

(2) In 2003, Barabási, as a guest of Collegium Budapest, was granted access to a database that contains the web browsing patterns of visitors of the Hungarian internet portal Origo.hu (of course, without any information on the personal identity of these users). This, at the time, comprised $40 \%$ of Hungary's internet traffic, adding up to over 6.5 million hits per month. Barabási began to examine the database with two young members of his research team. They also, in a certain sense, came up with negative results. Drawing on the hypothesis on the random transformation of one element into another (Ernest Rutherford's laws of transmutation) as an analogy for their

6 The notes at the end of the book contain references to the articles from scientific journals based on which Barabási builds his argument. 
experiment, they found that the number of potential readers for individual articles - contrary to their expectations - did not show a rapid decline and, thus, did not follow the exponential pattern as prescribed by the Rutherford's laws of transmutation. Their research report was published in 2006 in Physical Review under the title "Dynamics of Information Access on the Web."

(3) The failure - at least from the perspective of science - of the above mentioned research projects are thought-provoking on two counts: (i) scientists have never examined such large databases in conjunction with human behavior, (ii) the point of consideration for the examination and the hypotheses were, in both cases, based on scientific analogies. Since, however, both studies yielded results that were in direct contradiction to the expected outcome, doubts are legitimately raised. How constructive is it to rely on findings from the natural sciences when undertaking social scientific research? Is it really futile to analyze phenomena belonging to the domains of such different areas of science along similar principles? Are social scientists who argue against the existence of universal laws in their field of investigation right after all? Is it, indeed, naïve to hope that one day we may articulate precise predictions? Barabási did not give up. He continued his search for proof.

(4) A book entitled Statistics of Deadly Quarrels (1953) by Richardson, who has already been mentioned as the pioneer of weather forecasting, contains a statistical analysis of wars and conflicts which took place between 1820 and 1949. He clearly established that the number of victims showed strict mathematical correlations: there were many conflicts that demanded a small number of victims, but there were only a few wars that resulted in a large number of deaths.

(5) Vilfredo Pareto, Italian economist and sociologist, had made a similar observation earlier in reference to the distribution of wealth: there are many poor people and relatively few individuals who are extremely wealthy or pull in an outstandingly huge income. What's more, the wealthy are significantly richer than a random distribution of wealth could ever explain ${ }^{7}$. In both of

7 In Linked (p.71) Barabási refers to this concept of Pareto as the so-called " $80 / 20$ rule." Pareto, as an avid gardener, noticed during harvesting peas that $80 \%$ of peas grew in $20 \%$ of the pods. Later, he observed similar ratios in conjunction with economic phenomena. For example, $80 \%$ of the agricultural land in Italy is owned by $20 \%$ of the population. What is now known as the Pareto Law has since been shown to manifest in a number of areas. For example, $80 \%$ of company profit is produced by $20 \%$ of the employees and $80 \%$ of organizational decisions are made in $20 \%$ of the meetings. Barabási observed similar ratios in relation to the internet, when looking at links, scientific references, and the social networks of Hollywood actors. 
the latter cases, we see the power law at work; "many small events coexist alongside a few extraordinarily large ones" (p.102). That is to say: in both cases, we can preclude the kind of randomness that would be reflected by a Poisson distribution of the events.

(6) In observing randomness in the popularity of web pages, Barabási discovered a similar pattern in 1999. An analysis of e-mailing habits produced the same correlations: instead of writing letters in a random manner, e-mail users are periodically extremely active and then cease their activities for a while. Thus, the distribution of sent e-mail messages also demonstrates the power law in every single case. Following a review of similar empirical observations of human habits, Barabási draws his general conclusion:

"No matter what human activity we examined, the same bursty pattern greeted us: long periods of rest followed by short periods of intense activity [...] Bursts were everywhere in nature, from the edits individuals published at Wikipedia.org, to the trades made by currency brokers; from the sleeping patterns of humans and animals, to the tiny moves the juggler makes to keep his sticks in the air. It was no longer e-mail or Web browsing we were studying; we were witnessing something deeply linked to human activity, something announcing loud and clear that none of us behaves randomly, ever. In and of itself, this was not surprising, as no one really believes he or she is ruled by chance. We all have free will, which complicates everything [...] Yet no matter what we did, we unconsciously followed the same law - a power law. Conceptually simple, yet rather surprising” (p.105).

(7) For Barabási, the bursts in various types of human behavior originate from prioritization. As we are incapable of simultaneously undertaking many tasks, we usually set up an order of importance and act based on this. He mentions the story of Charles Michael Schwab, for instance, who was manager of the first million-dollar conglomerate in the world during the first decades of the twentieth century. Schwab significantly increased the productivity of various jobs through a very simple method: he asked employees to prioritize their tasks for the following day and perform their work accordingly. They were told to cross out the completed tasks and continue with the next item on their list. If they ran out of time, they were to carry the remaining tasks over into the next working day. Today, this simple approach serves as the basis of most manuals on time management. Famous nineteenth century French mathematician and physician Siméon Denis Poisson - to whom the author makes a number of references throughout the book - also used this method. 
He recorded his new ideas in a small notebook and only started on a new task once he had finished the previous one. New ideas were realized in the order suggested by the priority of the problem. In light of all this, Barabási meticulously analyzed the effect of prioritization in human behavior and came to the conclusion that the distribution of "waiting time" for tasks (the length of time tasks "wait" on our list before they are completed) arranged in an order of importance follows the power law.

(8) Barabási was also interested in finding out whether bursts were merely a result of the electronic age or if they were detectable in earlier periods as well. He found his answer while studying the correspondence of Albert Einstein and Charles Darwin. In his co-authored paper "Darwin and Einstein Correspondence Patterns" published in Nature in 2005, he showed that the correspondence attempts of these two geniuses were showing patterns that were hauntingly similar to those observed in e-mailing habits ${ }^{8}$. Based on this, it seems clear that the power law and bursts which can be observed in human behavior significantly predate the spread of technologies. Could it be that they reflect universal principles of human activity? Or could it be that this is about something even bigger?

(9) Meanwhile, biologists have confirmed that the movement of animals, especially during their search for food, shows a similar pattern. With the help of small detectors the flight patterns of albatrosses - the largest birds in the world - were analyzed to show that their fishing habits followed the familiar pattern. From spider monkeys to moose, bumble bees, fruit flies, and grey seals, the same universal scheme of mobility was found. Barabási begins to use the notion of Lévy flight, which relates closely to the concept of the power law, while introducing this research ${ }^{9}$. In physics, random walks have a number of categories:

(i) in the case of regular random walks, the randomly-moving object travels the same distance with each jump, the length of which follows a Gaussian distribution (for example, the atomic trajectories studied by Einstein, which have already been mentioned);

(ii) in case of a Lévy flight, however, we observe erratic movement with the length of the jumps following the power law. In other words, we see a random combination of many tiny steps and periodic big jumps.

8 In Barabási's analysis, only the size of the exponent was shown to be different, which reflects the variety of communication technologies through the ages.

9 The phrase refers to twentieth-century French mathematician Paul Lévy. 
(10) The notion of Lévy flight set off yet another scientific landslide, and the theory today points far beyond animal behavior research. In cell biology, for example, according to recent analyses, the protein that is responsible for controlling human gene activity is also linked to the DNA strand in this manner. Information stored in human memory is also retrieved by similar means. In fact, our eyes also engage in Lévy flight, when they encounter a new image: we "palpate" large images which cannot be perceived all at once by circling individual details with small eye movements and then jumping to another distant spot to once again repeat the exploration with tiny adjustments. Based on this observation, Barabási points to "nature's frugality and its tendency to apply the same solutions in quite different settings" (p.163). Nevertheless, if human behavior did actually reveal patterns similar to a Levy flight, people would never - or only by accident - manage to make their way home. This paradox inspired new research.

(11) Linked also sparked significant interest among mobile service providers. One of the companies asked Barabási and his research team to map mobile social networks and entrusted the team with the anonymized data of hundreds of thousands of telephone calls. Their results showed that there was a strong correlation between the behavioral habits and social networks of mobile users. The database, however, also provided a means for effectively tracking the whereabouts of callers, as it also included information about the location of the tower in whose reception area the calls were made. Barabási and his colleagues, through analyzing part of the data, also confirmed that human mobility showed a unique pattern: most callers only travelled a few kilometers between calls, while a few covered hundreds of kilometers. The distances followed the power law. As more and more data was processed, however, new doubts surfaced about the validity of the Lévy paradigm in reference to human behavior.

(12) Doubts were further fueled by a discovery in 2007 which revealed that the Lévy pattern observed in conjunction with the migration of the albatross was the result of an unfortunate error in measurement. Nevertheless, in spite of this glitch, the 1996 report proved to be remarkably productive in that it inspired the publication of hundreds of scientific articles. In these, the Lévynature of food gathering behavior in animals was shown for additional species (sharks, bony fish, sea turtles, penguins, etc.).

(13) The above doubts were somewhat eased by a reinterpretation of the movement of the dollar bill phenomenon. As the movement of banknotes does 
not reflect the movement of individual persons per se, banknotes following a Lévy flight pattern do not imply that people also move in this pattern. Further analysis of the mobile data indicated that the movement of most people is limited to a few well-delineated locations.

Barabási and his colleagues also measured the radius of movement for mobile users. Once again the power law pattern emerged. The theory that exceptions - in this case, a few individuals moving in very large circles - are part of the rule was also confirmed. Dollar bills follow the Lévy pattern because there are a few individuals of exceptional mobility who periodically carry the banknotes large distances. We, humans, however, do not move in accordance with this trajectory, so we have a good chance of finding our way home.

(14) In recent years, a number of studies have examined the movement of university students in the United States with the help of wearable computers and smart phones. In 2004, for instance, such data was collected on the mobility of a hundred student volunteers at MIT for the duration of one year. The analysis of 450,000 hours worth of data yielded shocking results. Based on the data, the movement of students could be predicted with a 9096\% probability. Barabási himself also underwent such an experiment: he wore a GPS device for months while a computer science student precisely followed and recorded his actual movement. After a few days, the student found Barabási's whereabouts to be predictable with $80 \%$ accuracy. In order to interpret these results, physicists rely on the concepts of entropy and redundancy. In the language of physics (or information theory), zero entropy signifies $100 \%$ predictability. If, for example, we were able to predict someone's movements at all times with total accuracy, his or her entropy would be zero. The other extreme is represented by those whose mobility, thanks to their maximal entropy, is completely unpredictable. The bursts that characterize their movements, as was demonstrated by the aforementioned studies in a number of areas, seem to make prognosticating their behavior with any accuracy exceedingly difficult. To put it another way: our entropy is not zero, but if we were able to precisely determine the degree of entropy, we would also be able to establish the degree of our unpredictability. Chaoming Song, a postdoctoral research associate working in Barabási's lab during his analysis of the mobile telephone database, worked out a procedure - based on the concept of redundancy - that can precisely estimate the actual entropy of individuals.

(15) Since most people's entropy is not zero, their movement is characterized by some degree of uncertainty. As there is already enough evidence at our 
disposal on how our various activities follow the power law, Barabási and his team were also expecting there to be considerable discrepancies in the predictability of activities. A new analysis of the mobile phone database, however, produced surprising results: an average predictability of $93 \%$ was found for all users. In the case of users with very low entropy, a predictability of nearly $100 \%$ was observed. At the same time - rather surprisingly not a single individual from the masses examined had less than an $80 \%$ predictability rate. The significance of this discovery is summarized by Barabási as follows:

,there is a fundamental difference between what we do and how predictable we are. When it comes to things we do - like the distances we travel, the number of e-mails we send, or the number of calls we make-we encounter power laws, which means that some individuals are significantly more active than others. [...] This also means that outliers are normal [...].

But when it comes to the predictability of our actions, to our surprise power laws are replaced by Gaussians. This means that whether you limit your life to a two-mile neighborhood or drive dozens of miles each day, take a fast train to work or even commute via airplane, you are just as predictable as everyone else. And once Gaussians dominate the problem, outliers are forbidden, just as bursts are never found in Poisson's dice-driven universe. [...] Despite the many differences between us, when it came to our whereabouts we are all equally predictable, and the unforgiving law of statistics forbids the existence of individuals who somehow buck this trend" (p.199).

(16) The predictability of the future is based on our knowledge of the past. This cliché-like statement is certainly supported by Barabási's research results. If I possess information - of sufficient precision and detail - on your whereabouts in the past and the degree of your entropy, I can predict at the given level of reliability, where you will be in the future. In this way, an indepth exploration of the past will get us closer to forecasting human behavior. The more reliable and extensive our data about past actions, the more we can expect our predictive ability to improve. Uncovering the past and generating databases on previous behaviors is no easy undertaking. We only have limited access to detailed databases of information on our past behavior, even in spite of a significant recent increase in the number of these databases. These limitations, rather than pointing to theoretical obstacles in the way of the calculability and predictability of the human future, however, draw our attention to the difficulties of practical implementation. In Barabási's words: 
"If we are not aware of the past, the future is hard to foresee. And what if our past suddenly becomes transparent? Our future, both as individuals and as a society, may cease to so mysterious. So, in order to reach into the future, we must first go back in time" (p.205).

(17) Medical research on various types of diseases has yielded many interesting results, which pertain to our discussion. These include the so called widow effect - where elderly spouses tend to die shortly after the passing of their partner - and the significant connections between social networks on the risk of obesity. According to the shocking results of research on the latter topic, if one of our friends gains weight, this increases our own chance of putting on weight within the next $2-4$ years by nearly $60 \%$. If this person is our best friend, this risk jumps to more than $170 \%$. Another study examined the timing of illnesses. In analyzing data on doctor's visits for two million patients over the age of 65, Barabási's team showed that the medical histories of the studied individuals were not random in the least. This is where bursts make their appearance: the occurrence of illnesses - or, to be more exact, the timing of the visit to the doctor - shows the same pattern as our web browsing or e-mailing habits. But what could be the reason behind this? Could it be our priorities - as in the case of the previous phenomena? It would be easy to answer with a quick no. The data nevertheless suggest that prioritizing also plays a role in visits to the doctor's office. Most people do not immediately turn to a physician upon experiencing mild symptoms; seeking medical help at this stage is lower on the priority list. But if the symptoms grow stronger, there is a significantly increased chance that the person will seek medical help. And this - as we have seen - prompts the e mergence of a burst like pattern.

(18) Japanese researchers used accelerometers attached to the body to show that human movement, including the finest wrist movements, also has bursts and that the length of immobile periods of rest follows the power law in all human beings. A portion of the subjects in this experiment suffered from severe depression. In their case, the periods of rest were on average more than twice as long as was measured for healthy individuals. Through this method, it is relatively easy to diagnose depression today. Biologists studying the operational mechanisms of cells have reached a similar conclusion. According to this, the activity of genes, proteins, metabolites and RNA molecules is not random; it is characterized by periodic, random bursts of activity. Regarding both evolution and the development of knowledge, Barabási observes that: "evolution proceeds in bursts..." (p.240). He then draws his final summarizing conclusion: 
"The phenomena we encountered in the previous chapters, from e-mail usage to travel patterns, hint that burstiness is deeply linked to human will and intelligence. Prioritizing only reinforces this impression, since it is our preferences that determine whether an action item is seen to immediately or indefinitely postponed. This would suggest that bursts require the ability to set priorities. But from this perspective, the results discussed above are rather humbling. They indicate that burstiness is not something we invented but was in use well before intelligent life ever emerged on Earth. There's nothing smooth or random in the way life expresses itself, but bursts dominate at all time scales, from milliseconds to hours in our cells; from minutes to weeks in our activity patterns; from weeks to years when it comes to diseases; from millennia to millions of years in evolutionary processes. Bursts are an integral part of the miracle of life, signatures of the continuous struggle for adaptation and survival" (p.240).

Albert-László Barabási, in his new book, gives readers something to ponder - especially social researchers who were previously unfamiliar with the research findings here discussed from the field of the natural sciences. Not to even mention the fact that empirical social scientists have not yet attempted a quantitative analysis of such immense databases. Barabási gives a detailed and fascinating account of these divergent, interdisciplinary studies. What's more, he does this at a time when it is evident to most social scientists that human behavior does not operate according to the universal laws that would explain the operation of social, economical or political systems. As for the possibility of prognosticating, there is very little or no discussion. If they consider the provision of explanations a task for social scientific research at all, they stress the role of social mechanisms, which, at best, help to show the frequent concurrence of easily identified schemes of behavior ${ }^{10}$. In contrast, Barabási offers the hope of Auguste Comte's and Karl Marx' dreams coming true. For if we are in possession of (a sufficient quantity and quality of) information about the past, we can discover universal laws based on which we can predict the future even in terms of human behavior ${ }^{11}$. All this said,

10 For a detailed elaboration of this viewpoint see, among others, P. Hedström - R. Swedberg (eds.): Social Mechanisms. An Analytical Approach to Social Theory. Cambridge University Press, Cambridge, 1998, and P. Hedström - P. Bearman (eds.): The Oxford Handbook of Analytical Sociology. Oxford University Press, Oxford, 2009.

11 In his book A General View of Positivism (which was originally published in 1844), Comte writes, "in politics - just as in astronomy, physics, chemistry, and physiology - observing the past can reveal to us the future." (Magyar Helikon, Budapest, 1979, p.51). Marx, in the preface 
it is true that, for the time being, he "merely" talks about human - and not social - dynamics. But let us not forget Comte, who in the middle of the nineteenth century also reasoned that "we cannot accept a single law of social development - even if its existence is confirmed by the historical method with the greatest authority - without rationally tracing it back to the positive theory of human nature"12.

Barabási's conclusions hit social researchers out of the blue - or, at least, incite a dispute. Counterarguments, however, must be thoroughly gathered and deeply thought through, as we are at a crucial turning point. This critical juncture is described by the author as follows:

"the quest to unravel human dynamics is more than an intellectual exercise. It is the last pillar of science, and its impact may one day prove to be on par with the physics of the early twentieth century or the unfolding revolution of genetics" (p.257).

Barabási's book is definitely worth reading and his argument is certainly worth carefully reflecting on. Because it may very well be that those who dismiss Bursts with a wave of their hand miss out on the most important development in the scientific progress of the twenty-first century.

\section{A GRAVE MISTAKE}

Before embarking on the critical evaluation of what are, for us, the most important thoughts in the book, we must get an extremely uncomfortable point of criticism out of the way. Barabási keeps returning again and again to the question of evaluating Count Telegdi's (an important figure in the Dózsa Revolt) prophecy on the Crusades giving way to a peasant revolt. Barabási refers to this "prediction" in asking whether it is possible to foresee the course of social phenomena. He disagrees with Popper's thesis on the predictability of social phenomena:

to the 1867 edition of Das Kapital refers to the movement of society as a natural law: "My point of view [...] regards the development of an economic social formation as a natural process." (MEM 23. Kossuth Könyvkiadó, Budapest, 1967, p.9). They both represent the approach of scientific naturalism, which was characteristic of the nineteenth century. According to this, the methods of natural and social science are - roughly - identical ("methodological monism").

12 The quote is taken from Auguste Comte's work entitled Cours de philosophie positive (18301842), in Hungarian: László Bertalan (ed.): Szociológiatörténeti szöveggyüjtemény [Reader in the History of Sociology.]. Manuscript, 1996, p.16. 
"Karl Popper proclaimed with thundering authority that 'for strictly logical reasons, it is impossible for us to predict the future course of history'. That is, foreseeing our future is not merely difficult or shady. It is downright impossible. Well, Sir Karl, how did Telegdi do it, then?" (p.260).

How? Telegdi's prediction was based on a naïve, layperson's insights on human nature and society. He saw the future like we all do to some extent. "Don't play with sharp objects!" Why do millions echo this to their children all over the world? Because, based on our everyday experience, we can make predictions for the future: if we give children sharp instruments, sooner or later they will cut themselves. We have experienced this numerous times in the past, so we can extend this knowledge to the future. This prediction, however, is of an entirely different quality to the predictions discussed by Barabási. It is completely unfounded to draw a parallel between Telegdi's prediction and Popper's thesis. The latter, of course, can be repudiated, but not on the grounds that Telegdi could foresee how things would happen, so Popper was wrong. Telegdi's example is a little bit (though not completely) like that of a lottery winner who, after winning the jackpot, brags that he knew that they would draw his numbers. And it doesn't really matter what reasons he gives because he only ran out of luck. There were probably some among Telegdi's contemporaries who had similar thoughts on the "Dózsa issue" and there were others who thought otherwise. This is how it's always been, and this is how it always will be. There have always been - and there will always be - some who foresee the future more precisely than others. Politicians, economists, sociologists, demographers have been - and are - able to give us "predictions" for the "future".

It is difficult to understand why Barabási needed the Telegdi thread. It does not support his argument, and, in itself, the Telegdi thesis is, in our opinion, unsustainable.

In spite of the "Telegdi issue," however, the author has our highest regard for his reference to Dózsa in his book. It is commendable that in his scientificallybased work, in addition to his scientific thesis, he also directs the attention of the scientific community towards a key moment in Hungarian-Szekler history. This does great service to Hungary's image as a country. Barabási, through his fame, has done quite a bit for the notoriety of many other Hungarian scientists by co-authoring publications. This is a very praiseworthy thing. His book Linked has been translated into numerous languages. It was a true bestseller and this is probably what will happen to Bursts as well. For many, Barabási's book will be a first introduction to Hungarian history, to the Szekler people; 
this is where they will first read about György Dózsa and Tamás Bakócz. He should be thanked for this.

And now let's see what two stamp collectors can possibly say to a physicist about human mobility forecasting.

\section{DATA EXPLOSION AND PREDICTION}

The thesis of the book is centered on the computer - or, to be more exact, perfection of the human ability to calculate and analyze. This is so for two reasons. With the widespread distribution of computers and other digital tools, we can record and gather larger amounts - and more types - of data on the various phenomena of our lives (for example, about human mobility, activity, and many other things). On the other hand, highly developed computers are also capable of performing the kind of computationally demanding operations that are required for processing large masses of data. Two important conditions are fulfilled simultaneously: we have something to analyze and we have something to analyze with. But there is also a third basic condition: processing data also requires adequate theoretical models (which are equipped to capture regularities in the examined area in a relevant way) and techniques of analysis.

Thanks to this data explosion (or from an alternate perspective: sensor revolution), highly complex systems have also become analyzable and predictable. The history of weather forecasting provides the best example. The theoretical model and the computational technology have long been available, but, in the absence of the appropriate amount of data and adequate computational capacity, forecasting proved to be an unsuccessful undertaking for quite some time. With the emergence of sufficient data, along with computers powerful enough to process it, the precision and reliability of predicting weather conditions increasingly improved.

As an analogy to this, Barabási's conclusion seems obvious: as the data explosion of our times - when sensors are capable of collecting data from human beings - runs its full course, there is a chance that we may be able to predict human behavior. As such, we are indeed on the threshold of a new age. Whether the age of computational social science is approaching (as it appears more and more in articles ${ }^{13}$ ), whether the new science will be called

13 See for example: David Lazer, Alex (Sandy) Pentland, Lada Adamic, Sinan Aral, Albert Laszlo Barabasi, Devon Brewer, Nicholas Christakis, Noshir Contractor, James Fowler, Myron Gutmann, Tony Jebara, Gary King, Michael Macy, Deb Roy, Marshall Van Alstyne: 
human dynamics, whether we will find some other label (or perhaps, leave the old ones in place), is not that relevant at the moment. Barabási's thesis is important and true, and the shift he captures and describes is of significance. We stamp collectors must also set foot on the path that physicists have set off on. And we must be able to share the knowledge - both in terms of theory and modeling - that we have thus far accumulated on human actions, social networks, groups and organizations, as well as institutions, social systems and subsystems.

\section{THEN WHAT ARE WE EXAMINING EXACTLY?}

The first important task is to precisely record what we want to collect data on, what it is we want to analyze and predict. In other words: in what, how and why are we trying to find regularities? On this question, Barabási is rather sloppy. Sometimes, he wants to examine human actions:

"The closer we look at them, the more obvious it will become that human actions follow simple, reproducible patterns governed by widereaching laws" (p.11)

At other junctures, he speaks about human mobility:

"Dirk's work, however, offered us our first real glimmer of hope that one day we might infer the laws of human mobility" (p.33)

Then there are instances where he wants to understand human behavior:

"what disturbed me was that [...] our understanding between the predictions and the measurements meant that our understanding of the Web-visitation process was inadequate. Grossly inadequate, in fact. And in turn, it also meant that our comprehension of human behavior must be seriously limited" (p.50)

Human mobility, behavior, actions, social actions - the concepts keeps changing, as if they were interchangeable. It may seem like splitting hairs, but we must insist: in analyzing social actions, it is necessary to precisely distinguish between these concepts. Human behavior does not only consist of mobility. What's more, there are certain human movements we do not consider behavioral. If a person, upon seeing an accident, just stands there and

"Computational social science", Science 323 (volume 5919), pp.721-723 (February 6, 2009). 
doesn't do anything to help those who might need it, we consider his or her immobility an action (which might even be punishable by law). Sometimes we see the blood pulsing in someone's artery, we see people's chests rising and falling as they breathe, but we do not consider these movements to constitute behavior. We don't ask these people "why are you moving the blood in your arteries?" or "why are you breathing?" But, in certain circumstances, we may ask someone "why did you touch my shoulder?" It is nevertheless true and important that we may discover regularities in any of our movements. Let us think here of the finding, also referred to by Barabási, according to which depression is preceded by a slowing down of body movements. As today this phenomenon is observable with detectors, this knowledge can be utilized towards prevention. But there are also other examples. To prevent truck drivers from dozing off at the wheel, eye-movement detectors are sometimes used to sound off an alarm in response to specific movement patterns that occur immediately before falling asleep, which helps keep the driver awake. In some cases, the engine may be turned off, so that the driver is forced to stop. Japanese researchers developed a new technology for "encrypting" mobile phones, where, instead of pushing buttons to unlock the phone, it is enough to shake it. As it turns out, everyone shakes their hand in a different, completely unique (inimitable) way. If the mobile phone "learns" this distinctive series of movement, only its owner can access the information stored on it. Subsequent (and perhaps even more surprising) discoveries on the regularities of human movements are sure to follow. But however important these discoveries and the new technologies that are based on them - are, we must recognize that human activities cannot be narrowed down to mere movements. If we wish to understand the specifically human features of mobility, we must also capture the intentionality and intended meaning of behavior, for it is only then that we can speak of human action. Action is a form of behavior that has intended meaning attached to it. It is for this reason that breathing per $s e$ is not considered an action in itself, as, in everyday life, people breathe without giving it meaning or intentionality. It can sometimes happen, of course, that we attach some kind of intention to our breathing, such as in the case of athletes or singers who consciously try to control their breathing, or the communicative sigh of an actor. In the general sense, however, breathing as a behavior is characterized by an absence of intention. Regardless, we can still, naturally, examine the regularities of breathing as a subtype of human behavior, but we do not try to find any attached meaning. But we can go even further in clarifying the concept, if, within actions, we distinguish social actions, and within that: the category of social relations. The intended meaning of social action is aimed at the behavior (and not action!) of another person. 
When the doctor gives medicine to a patient in order to regulate his breathing, then the subjective meaning of her action is the intention to heal. Since the doctor wants to cure the patient, we think of this as a social action. We can talk of a higher degree of the social aspect when at least two persons of social action mutually adjust their own actions in order to harmonize them. It is at this point that we talk about a social relationship. Based on all this, we are ready to outline a somewhat sketchy - but seemingly useful - typology, which distinguishes the following concepts and levels: i) human mobility, human behavior; ii) human actions; iii) social actions; iv) social relationships. These important and fertile distinctions by Max Weber were further deepened by the analytical action theory of the twentieth century, which includes rational choice and game theories ${ }^{14}$.

The meaning and point of this distinction is to grasp intentionality. The scientific exploration of phenomena moves from description towards explanation/comprehension (and later prognostication). When looking at action, it is important to consider the duality that is inherent in Weber's concept of "understanding the intended meaning." Weber talks about two kinds of understanding, both of which are equally important when studying actions.

Direct understanding, to Weber, is when we can describe phenomena "in themselves," in the way they affect one another and follow from one another. Someone is running after someone else. We can see this and we can describe how it is happening. The "mere" description of things takes place in a similar manner in all areas of science. In such cases, we can even find repeating, typical patterns (for example, those of the power law) in the examined phenomena, just as it happens time and again in Bursts. The other level of understanding action is that of motivational understanding, on which the causal (or intentional) explanation of the action is based. Someone is running after someone else because they are racing each other, or because one is chasing the other. When we are analyzing actions - especially social actions - motivational understanding is required for the mapping and interpretation of the intended meaning.

Bursts "doesn't care about" the above distinctions. And, perhaps for the time being, they are not needed. While the accumulation of knowledge is the primary aim of a given, new field of science, explanations can be put off till later. There is still enough to be surprised about when we see how often bursts appear (the power law manifests) in revealed patterns. While there is tremendous potential

14 For his brief review of action theory: Syi: Cselekvéselmélet dióhéjban [Action Theory in a Nutshell], Typotex, Budapest, 2008. 
in computerized pattern recognition, we must know that interpretation and explanations cannot - for the time being - come from machines, only from human beings. And pattern recognition in itself is not worth much if we don't have some kind of an explanation to go with it. We would like to demonstrate this point with a hypothetical example. One of us has discovered that the odd and the even chapters of Bursts are more closely related to one another, while another one of us has counted how many pages there are in the even- and oddnumbered chapters. We have come to the conclusion that there is a significant difference in the distribution of page numbers.

Figure 1 Length of chapters (number of pages)

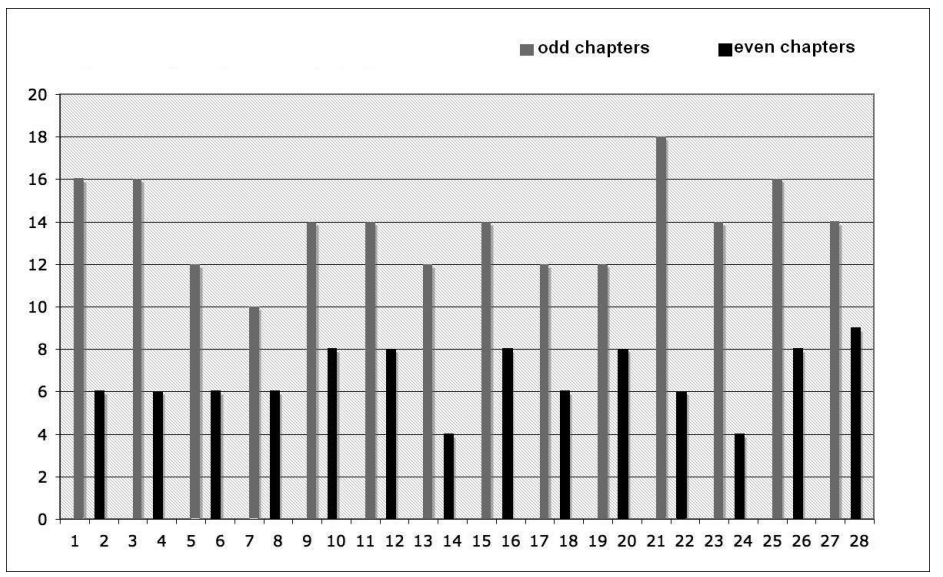

The average length of the even chapters (which recount the story of the Dózsa Revolt) is 7 pages in the Hungarian edition. This number is 14 for the odd chapters. It is a pattern, alright, even a surprising one at that, but let us admit: the recognized pattern is of little use or interest to us. So, until we know more about the author's motivations, the recognized pattern has no meaning and there is nothing we can do with $\mathrm{it}^{15}$.

It is no accident that when Barabási talks about the real reasons behind the Crusades, he pinpoints the power struggle between the Pope and Cardinal Bakócz as a central element. Pattern or no pattern, social actions can only be validly described and explained through motivational understanding. In the

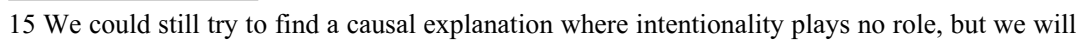
not discuss this possibility here. 
aforementioned example, this is what Barabási does as well - from instinct. From this point on, it is precisely this that we must endeavor towards in a conscious, reflective manner.

We can also find regularities in the world of social actions if we take intentionality into account. Patterns form in our lives because i) we have become used to certain forms of behavior, and ii) we prescribe social norms for ourselves and others and perform actions that conform to these. Once we have uncovered the patterns behind our actions and we are also curious about the potential explanations, we must try to discern the habits, interests and norms behind the patterns. Having noticed, for example, that the retired pensioner at home, the prisoner in the prison, the soldier in his barracks, and the farmer on the farm all wake up at 5 A.M., we will probably find very different meaning and actions behind the shared pattern. The reason for waking at this time will be habit for the retired pensioner, enforced regulation for the prisoner, duty for the soldier and self-interest for the farmer. It is all the same - and still different.

In the kind of human-related phenomena and patterns shown in Bursts, motifs are still difficult to discern today, and in some cases they are even missing. But we also must note that Barabási's examples have to do more with human mobility and behavior than human actions. For this reason, we also take advantage of Barabási's weather forecast analogy: why couldn't we refer to the prognostication of human behavior as human mobility forecasting?

\section{LEVELS OF ANALYSIS}

We have previously quoted Barabási's point about physicists being able to predict the pressure and temperature of a gas (which is composed of molecules) without the ability to foresee the trajectory of individual gas molecules. When he uses this as an analogy in reference to human behavior, he is suggesting that regularities can only be found in human behavior at the social level. This suggestion is seemingly contradicted, however, by the fact that he detects the power law not only in the e-mailing habits of millions but also behind the behavioral patterns of individual people (for example, Darwin's correspondence).

"The same is true for human dynamics. Our deep-rooted unpredictability does not need to bubble up at the level of society. If we carefully distinguish the random from the predictable, we might be able to foresee many features of the social fabric" (p. 256). 
As he is not a social scientist, Barabási builds his argument without reflecting on the differences between the various levels of social analysis. Consequently, in his examples, things that require distinction are mixed together. In examining social phenomena, we usually draw distinctions between the approaches of methodological individualism and methodological collectivism. As a classic example of this, we can refer to the fields of micro- and (traditional) macroeconomics, which are markedly distinct but mutually determine each other. The individualistic perspective in explaining macro-level economic and social phenomena refers to the individual in action and searches for casual connections between collectivistic "supra-individualistic" phenomena. While both can serve as a source for new knowledge, we must be careful not to confuse the levels of analysis. Economic and social phenomena at the macro-level cannot be explained without an exploration of the mechanisms of action that connect the macro- and micro-levels. This reflection does not take place in Bursts, though it would be sorely needed, especially in its endeavor to find an explanation for the power law - a pattern observed in such varied phenomena. This absence and non-reflection cannot really be held against Bursts, however. The real virtue of the book is in its recognition of similar patterns in different phenomena. Explanations can come later. When we talk about war, the economic crisis or an epidemic, we can make our observations from the perspective of methodological collectivism. When we look at habits of letter writing, e-mailing, or travelling, we should assume an individualistic approach. Finally, when we are examining patterns of vision (visual palpation) and hand movements, we are no longer really looking at social phenomena. While patterns of the power distribution can manifest in all of these scenarios, the explanations can be found in different places.

\section{OTHER FORECASTS}

Without wishing to trivialize what Bursts has achieved, we must note that it is somewhat unfair and misleading to suggest that we are just now arriving to a moment in history where we are given a chance to uncover the general regularities of social phenomena. Well-established prognostication systems that describe and predict - sometimes with great accuracy - the laws of human and social behavior have been in place for quite some time.

Demography has been able to predict population mobility with a high degree of reliability for many years, even if demographers are sometimes wrong. Based on data taken from representative samples of public surveys, we have been able to predict the distribution of opinions for the entire population for 
decades. Geospatial information analysis is a long known and used method of pattern recognition. Geospatial information systems are capable of predicting spatially segmented economic, political, cultural, and consumption patterns through an analysis of sources and data that are topologically unevenly distributed in geographic space. For simulating changes in social relations, evolutionary game theory applies analytical apparatus that can forecast the selection and propagation - through learning and social mimicry - of action and assessment strategies.

We can also find analysis within the digital universe, however, that appeared long before any research on human dynamics. Data mining uncovers the "hidden" patterns of large databases and has been applied successfully in analyzing bank, telecommunication and financial databases. While different methods (for example, clustering) are employed to recognize hidden correlations - and the power law is not the only thing that it detects -, data mining is similar in its "logic" and productiveness to the methods discussed in Bursts.

In the fairly early days of popular web use, so called recommender systems and collaborative filtering systems already existed. Based on data on netrelated human activities (reading, visiting, purchasing, voting), these systems could not only cluster users with similar tastes/values and generate user profiles, but could also provide group members with (often very reliable) predictions. These recommender systems, based on the analysis of events and actions (clicks, purchases, views, votes, etc.) that reveal previous value commitments, can make suggestions for the "future," which have a high probability of being accepted by the addressed users. The Amazon web store and LastFM online radio stations both work in this manner. What is this is if not predicting the future?

\section{WHY THE POWER LAW?}

Throughout Bursts, the power law plays a key role ${ }^{16}$. It is indeed fascinating how "many places" the patterns and regularities of the power law can be

16 Competing concepts and theories exist in reference to the same problem not only in the social sciences but in the world of physics as well. In place of the "power law, the mathematical signature of bursts" (p.124), one of the central concepts of the book, Barabási - as well as others in other places - also uses the term "scale-free". Elsewhere, the same phenomena is also called Zipf's law, Pareto distribution, Pareto principle, Levy flight, the 80/20 rule, and long tail distribution. We can also list fractals from chaos theory here. 
observed $^{17}$ - in the dimensions of craters, the size of human settlements, the frequency of certain words, eye movements, in the mobility of animals on the search for food, in the world of cells and molecules, in our actions of communication, in our travel habits, and we could go on. For a social scientist, however, Barabási's suggestion that the assertion of some type of priority is required before the power law (at last in part) can manifest is even more exciting.

"This would suggest that bursts require the ability to set priorities" (p. 240)

When examining the distributions of correspondences, e-mails, and diseases, Barabási attributes explanatory power to the establishment of priorities, but without further elaborating on this point and seeing where it leads. It would be worth doing, even if the author is aware - and in fact asserts - that, in the case of many natural and social phenomena (for example, the size of craters or settlements, the frequency of certain letters) it would be difficult to attribute real explanatory capacity to priorities in reference to the appearance of the power law.

Nonetheless, after reading Bursts, one of the most inspiring questions for a social scientist is: to what extent can we accept, and how can we interpret, the central role and explanatory potential of priorities (or preferences) when analyzing the burst patterns of social phenomena. This is an exciting and, so far, unanswered question. At a certain point in his book, Barabási - more intuitively than consciously - but that is beside the point here - "hides" a few concepts that are closely related to the basic categories of Weber's action theory (which is so dear to us):

"Humans are not that different from pollen suspended in water. Driven for reasons that are just as enigmatic as the pollen's motion, we display a ceaseless desire to move most of the time. We are not kicked by tiny, invisible atoms but dragged by the imperceptible flickering of our neurons, which we translate into tasks, responsibilities, and motivations" (p.28).

When explaining actions, the most fundamental categories are desires and possibilities, intentions, beliefs, preferences, and will, from which we can derive other key categories, such as value, power, domination, order, normalcy, organization, institution, and so on. Barabási is scratching at "the right" surface. Now let's start digging.

17 M. E. J. Newman: "Power laws, Pareto distributions and Zipf's law." Contemporary Physics 46 (2005), pp.323-351. 


\section{SOCIAL FACTORS}

If we are to get to work on this, however, we must take everything into account. We must consider what, perhaps, the physician sees most clearly: the facts. If we have tons of data, then let's analyze it the way it should be analyzed. But let us also not forget: we are looking at society, at people. Thus, social factors must also be brought into the analysis.

"It makes you wonder, do humans follow hidden laws, laws other than those of their own making?" (p.7)

Barabási, in this book, has shown us that there are hidden regularities in human mobility and behavior, which can be identified and even predicted. This is a great lesson for us, stamp collectors. But as philatelists, we also see what Barabási doesn't handle according to its weight. This is not to say that he has no awareness of it. He even calls it by name when he states that, in a person's life, self-made rules are just as important. To be sure, we can also collect data, make calculations and search for patterns on this terrain. But we will also need to understand and explain how and why human beings follow their self-made laws. Barabási's proposition: "Think of yourself as a dreaming robot on autopilot, and you'll be much closer to the truth" (p.11). In our opinion, he is mistaken here. As human beings - at least in part because of our self-created laws - we can act in a reflexive manner in the world. We create culture around us. This is what makes us - and keeps us - human. 